\title{
A Popularização Diagnóstica do Autismo: uma Falsa Epidemia?
}

\author{
Maíra Lopes Almeida ${ }^{1}$ \\ ${ }^{1}$ Universidade Federal de Uberlândia, MG, Brasil.
}

\author{
Anamaria Silva Neves ${ }^{1}$ \\ ${ }^{1}$ Universidade Federal de Uberlândia, MG, Brasil.
}

\begin{abstract}
Resumo: Atualmente, aponta-se que a prevalência de crianças diagnosticadas com Transtorno do Espectro Autistaé de uma criança para cada sessenta e oito. Diante disso, aventa-se a possibilidade de uma epidemia. Este trabalho tem como objetivo tecer considerações a respeito do aumento de crianças diagnosticadas com Transtorno do Espectro Autista contemporaneamente. A partir de uma investigação teórica, procurou-se conjecturar hipóteses para esse fenômeno e suas devidas implicações para a prática clínica do psicólogo. Foram realizadas pesquisas em bases de dados como PubMed, Medline e Biblioteca Virtual em Saúde - Psicologia Brasil. Os dados epidemiológicos encontrados apontaram para um aumento significativo do diagnóstico nos últimos anos, o que provocou uma questão sobre essa possível epidemia. Esses achados nos fizeram supor que tal aumento se edifique pela articulação entre as perspectivas psiquiátrica e social. A discussão dessa hipótese sustenta, então, que a prática do psicólogo diante da demanda referente ao sofrimento na infância deve ser pautada por um posicionamento ético e por uma clínica atenta ao cuidado.
\end{abstract}

Palavras-chave: Autismo, Diagnóstico, Epidemia, Psicologia.

\section{The Diagnostic Popularization of Autism: a False Epidemic?}

\begin{abstract}
Currently, estimates point to the prevalence of children diagnosed with Autism Spectrum Disorder (ASD) of one child every sixty-eight. The possibility of an epidemic is considered. This study makes some considerations about the increase in children diagnosed with ASD in the last decades. As a theoretical investigation, we thought of hypotheses for this phenomenon and its implications for the psychology clinical practice and searched databases such as Pubmed, Medline and BVS-PSI. The epidemiological data found indicated a significant increase in diagnosis in recent years, which raised a question about this possible epidemic. These findings have led us to suppose that such an increase is built by the articulation between the psychiatric and social perspectives. Our hypothesis holds that the psychologist's practice for suffering in childhood should be guided by an ethical stance and a clinic attentive to care.
\end{abstract}

Keywords: Autism, Diagnosis, Epidemic, Psychology. 


\title{
La Popularización Diagnóstica del Autismo: ¿una Falsa Epidemia?
}

\begin{abstract}
Resumen: Actualmente, se señala que la prevalencia de niños diagnosticados con Trastorno del Espectro Autista (TEA) es de un niño por cada sesenta y ocho. Esto sugiere la posibilidad de una epidemia. Este trabajo tiene como objetivo tejer consideraciones acerca del aumento del diagnóstico de niños con TEA contemporáneamente. A partir de una investigación teórica, se buscó conjeturar hipótesis para ese fenómeno y sus debidas implicaciones para la práctica clínica del psicólogo. Se realizaron búsquedas en bases de datos PubMed, Medline y Biblioteca Virtual en Salud - Psicología Brasil -. Los datos epidemiológicos encontrados apuntaron a un aumento significativo del diagnóstico en los últimos años, lo que provocó una cuestión sobre esa posible epidemia. Estos resultados suponen que el mencionado aumento se edifique por la articulación entre las perspectivas psiquiátrica y social. La discusión de esa hipótesis sostiene que la práctica del psicólogo ante la demanda con relación al sufrimiento en la infancia debe ser pautada por un posicionamiento ético y por una clínica atenta al cuidado.
\end{abstract}

Palabras clave: Autismo, Diagnóstico, Epidemia, Psicología.

\section{A popularização diagnóstica do autismo: uma falsa epidemia?}

De acordo com as últimas estatísticas, o autismo atinge atualmente uma em cada 68 crianças (Center for Disease Control and Prevention, 2014). Chama a atenção um diagnóstico de transtorno psiquiátrico da infância que cresceu vertiginosamente em um curto período de tempo (Rios, Ortega, Zorzanelli, \& Nascimento, 2015). Conhecido atualmente como transtorno do espectro autista (TEA), sua etiologia ainda é desconhecida e, embora se admita a multicausalidade, há diversas conjecturas sobre o que favorece seu surgimento. Com o aumento crescente de diagnósticos, as suspeitas se ampliaram e deram origem a diversas hipóteses não comprovadas.

Geralmente, este é um transtorno identificado ainda na primeira infância e implica possíveis impactos para os irmãos, alterações na dinâmica familiar, eventos estressantes e sobrecarga de cuidadores (Araújo, Souza-Silva, \& D’Antino, 2012; Fávero \& Santos, 2005; Gomes, Lima, Bueno, Araújo, \& Souza, 2015). Por meio de uma investigação teórica em bases de dados como PubMed, Medline e Biblioteca Virtual em Saúde Psicologia Brasil BVS-PSI, este trabalho tem como objetivo tecer considerações sobre o diagnóstico de TEA na atualidade. A confirmação do aumento de crianças diagnosticadas a partir das pesquisas epidemiológicas incita a conjecturar posicionamentos, questões e argumentos a respeito desta inflação.

\section{Espectro do transtorno autista: dados epidemiológicos e contemporaneidade}

Aparentemente, há um espectro que ronda a infância - o espectro do Transtorno Autista ${ }^{1}$. Eleito em janeiro de 2012, na França, como a grande causa nacional, o autismo se disseminou e hoje configura uma suposta epidemia. Passaram-se 73 anos desde seu isolamento por Kanner (1943/1997). Como mencionado, o distúrbio atinge atualmente cerca de uma em cada 68 crianças, segundo estatísticas norte-americanas (Center for Disease Control and Prevention, 2014).

Kanner foi o primeiro a estabelecer o autismo como um distúrbio único, e não mais como apenas um sintoma da esquizofrenia. Ao observar onze crianças, o autor pôde descrever o que chamou de distúrbios autísticos inatos do contato afetivo, sendo que esta, "até agora não descrita e bastante rara, parece, ... seria mais frequente do que a raridade dos casos indica" (Kanner, 1943/1997, p. 167). O autor caracteriza a síndrome como uma incapacidade inata e descreve como

\footnotetext{
${ }^{1}$ Menção à frase inaugural do Manifesto Comunista escrita por Marx em 1848 em que ele faz alusão ao espectro do comunismo que rondava a Europa. Fazemos uso da polissemia da palavra espectro, que como descrito nos manuais diagnósticos refere-se à representação de intensidade, enquanto na frase de Marx refere-se a um fantasma que se apresentava e assustava os europeus o que nos remete à situação dos pais diante do aumento de diagnósticos de autismo.
} 
sintomas os traços obsessivos, a estereotipia e a ecolalia. A pioneira caracterização do autismo por Kanner serve, ainda hoje, como referência para as definições encontradas nos atuais manuais diagnósticos.

A definição de autismo se ampliou no decorrer da história, sobretudo com a admissão do espectro, que o tornou, na $5^{\mathrm{a}}$ edição do Manual Diagnóstico e Estatístico de Transtornos Mentais (DSM-V) (American Psychiatric Association, 2014), "Transtorno do Espectro Autista" (TEA). A partir dessa nova nomenclatura, o autismo englobou o Transtorno Desintegrativo da Infância, Transtorno de Asperger e Transtorno Invasivo do Desenvolvimento Sem Outra Especificação.

O diagnóstico de TEA é realizado clinicamente com base nos critérios do referido manual, observações do comportamento e entrevistas com os pais e/ou cuidadores. É possível também realizar exames que excluam outras doenças associadas, como surdez (Ribeiro, 2007).

Com relação à tendência da ampliação de casos de TEA, pesquisas feitas sobretudo na Europa e nos Estados Unidos registraram um consistente aumento de crianças diagnosticadas como autistas nos últimos anos (Bertrand, Mars, Bove, \& Yeargin-Allsopp, 2001; Charman, 2002; Fombonne, 1999; Rice et al., 2012; Rutter, 2004).

O primeiro estudo epidemiológico sobre autismo consta de 1966, na Inglaterra, por Vitor Lotter (Ribeiro, 2007). Naquela época, ele encontrou uma prevalência de 4,5 crianças autistas para cada dez mil. Outros estudos também conduzidos entre o fim da década de 1960 e início da década de 1970 na Europa apontam para estimativas de um autista para cada 2.500 crianças (Center for Disease Control and Prevention, 2014).

Desde então, a prevalência global de autismo aumentou, aproximadamente, trinta vezes. As estatísticas da doença tornaram-se mais acessíveis, pois, no ano 2000, o Center of Diseases Control and Prevention (CDC) criou o Autism and Developmental Disabilities Monitoring (ADDM), uma rede que se ocupa das estimativas e prevalência de TEA nos Estados Unidos. A partir de sua criação, foram realizadas pesquisas periódicas, de dois em dois anos, que monitoram os dados epidemiológicos de autismo.

Por meio de estudos, o ADDM permite acompanhar a evolução dos diagnósticos da doença a cada biênio. Na primeira investigação, realizada no ano 2000, havia um autista para cada 150 crianças examinadas (uma prevalência igual a $0,66 \%$ ). Na segunda pesquisa, em 2002, o número se manteve. No entanto, a partir de 2004, os números se tornaram consideravelmente maiores, de forma que a última estimativa apontou um autista para cada 68 crianças, perfazendo a prevalência igual a $1,47 \%$.

De acordo com os dados divulgados pelo CDC (2010), o valor de prevalência de TEA em 2010 foi duas vezes maior que nos dois primeiros anos da pesquisa, 2000 e 2002. Segundo Fombonne (2003, 2009), pesquisas futuras deverão registrar um aumento ainda maior. Existem algumas projeções que demonstram que até 2050 haverá um aumento de 42,7\% em menores de cinco anos com TEA nos Estados Unidos, o que significa 76.000 crianças. A partir de suas revisões, o autor compreende que o autismo é muito mais comum do que pensávamos anteriormente.

Diversos estudos que registram o aumento da prevalência desses casos apresentam também limitações. Os assustadores números, devido a essas limitações, transportam em seu cerne a incerteza sobre a realidade dessas estimativas.

Em função das dificuldades operacionais face aos delineamentos epidemiológicos necessários para a aferição de incidência de autismo, a maioria dos estudos epidemiológicos realizados refere-se, então, à medição da prevalência. Como aponta Klin (2006), o aumento nas taxas de prevalência não significa um aumento da incidência. No entanto, para o autor, a crença sobre o aumento da incidência é a responsável pelo surgimento da ideia errônea sobre a "epidemia de autismo", noção que sequer se sustenta empiricamente, uma vez que os poucos estudos sobre incidência não foram adequados para testar a hipótese da epidemia (Fombonne, 2009).

É possível também questionar o aumento de prevalência, na medida em que muitos estudos entre as décadas de 1960 e 1970 associaram autismo e retardo mental. Assim, as pesquisas realizadas com indivíduos sem comorbidade começaram somente após algum tempo. Além disso, os diferentes métodos empregados nos estudos epidemiológicos alteram consistentemente os dados sobre a prevalência. Dessa forma,

estudos que dependem de fontes administrativas únicas para identificar casos produziram baixas estimativas enquanto investigação, utilizando métodos proativos para o encontro de casos, ou seja, múltiplas fontes de apuração e procedimentos 
diagnósticos diretos produziram taxas muito mais altas (Fombonne, 2009, p. 88, tradução nossa)².

Outra questão que surge a respeito das investigações epidemiológicas sobre o autismo refere-se às publicações em todo o mundo. Segundo Paula, Fombonne, Gadia, Tuchman, \& Rosanoff (2011), grande parte dos estudos sobre prevalência são realizados na Europa e Estados Unidos, sendo que muitos países não dispõem desses dados tão bem documentados, incluindo o Brasil.

A dificuldade no estabelecimento da incidência, o obstáculo na comparação desses indicadores ao longo do tempo e a lacuna sobre pesquisas epidemiológicas específicas para a realidade de alguns países tornam a "epidemia de autismo" questionável. No entanto, desacreditar a epidemia não significa desconsiderar o aumento, mas poder, livres de seus atavios, problematizá-la de tal forma a compreender seu fundamento.

Esse fundamento encontra um de seus amparos na dificuldade mais recorrentemente citada nas pesquisas epidemiológicas para o autismo: as mudanças de definição e classificação ao longo do tempo. Assim, "a menos que as comparações também controlem rigorosamente as mudanças nas definições do caso, as interpretações das diferenças nas taxas de prevalência ao longo do tempo e através de pesquisas serão virtualmente impossíveis" (Fombonne, 2009, p. 88, tradução nossa) ${ }^{3}$.

A definição de Kanner, considerada restrita, sofreu diversas alterações ao longo do tempo. As mudanças paradigmáticas nos manuais diagnósticos psiquiátricos afetaram a classificação e a nomeação do autismo. Essas modificações têm sérias consequências para os dados epidemiológicos. A partir da base psiquiátrica se funda a definição do transtorno que subsidiará as pesquisas de incidência e prevalência. Para tanto, a noção de epidemia no caso do autismo está fortemente associada às mudanças paradigmáticas ocorridas na psiquiatria no século passado.

\section{A perspectiva psiquiátrica: de sintoma a transtorno do espectro autista (TEA)}

Nos manuais psiquiátricos que embasam a realização do diagnóstico houve mudanças históricas na definição do autismo. Em 65 anos, o autismo transformou-se de sintoma das psicoses infantis em TEA, pelo DSM-V (American Psychiatric Association, 2014). Compõe a estrutura de sintomas os prejuízos nas áreas de comunicação social e padrões de comportamentos repetitivos e restritivos.

A história do autismo nos manuais psiquiátricos se inicia no DSM-I (American Psychiatric Association, 1952), como uma manifestação sintomática pertencente ao quadro da esquizofrenia em crianças. Na segunda versão deste mesmo manual, novamente ele é classificado como um sintoma da esquizofrenia de tipo infantil (American Psychiatric Association, 1968).

Na década de 1980, com a publicação do DSM-III (American Psychiatric Association, 1980) e sua revisão, o autismo infantil aparece como uma subcategoria da classe diagnóstica de Transtornos Invasivos do Desenvolvimento (TID). Com a chegada do DSM-IV (American Psychiatric Association, 1994), integram-se aos TID, juntamente ao autismo infantil, as subcategorias de Síndrome de Rett, Transtorno Desintegrativo da Infância, Transtorno de Asperger e Transtorno Invasivo do Desenvolvimento sem outra especificação. Na atualidade, todos esses transtornos, exceto a síndrome de Rett, foram fundidos em um único denominado TEA (American Psychiatric Association, 2013).

Bursztejn (2016) afirma que o autismo apareceu pela primeira vez em uma classificação oficial na 9a edição da classificação internacional da OMS, figurando em um capítulo referente às "psicoses específicas da infância”. Na década de 1980, com a publicação da terceira versão do DSM, o autismo passa a integrar os Transtornos Invasivos do Desenvolvimento. Assim, em menos de quinze anos, "a noção de 'Transtorno Invasivo do Desenvolvimento' substituiu a noção de psicose infantil, com um foco crescente no autismo" (p. 16). O autor argumenta que as classificações atuais se amparam em duas abordagens: categorial e dimensional. Enquanto na categorial é interessante que a população identificada com cada diagnóstico seja distinta, a experiência clínica constata sobreposições importantes nos TID. Isso provocou uma alteração, de forma que a abordagem dimensional mostrou-se estar mais em consonância com a realidade destes

\footnotetext{
${ }^{2}$ No original: "studies relying on single administrative sources for identifying cases yielded low estimates, whereas investigations using proactive methods for case finding, that is, multiple sources of ascertainment and direct diagnostic procedures, yielded much higher rates".

${ }^{3}$ No original: "unless comparisons also control rigorously for changing case definitions, interpretation of differences in prevalence rates over time and across surveys will be virtually impossible".
} 
transtornos por compreender uma mesma patologia, porém, com variantes mais ou menos severas. Nessa concepção, os TID são como um continuum, agora transformado em um espectro.

A mudança para uma concepção de espectro, oficializada com a publicação do DSM-5, amplia a imprecisão dos critérios de inclusão. $\mathrm{Na}$ opinião de Touati, Mercier e Tuil (2016), essa ampliação "parece, sob muitos aspectos, um depósito para quadros bastante heterogêneos no plano clínico, psicopatológico e etiopatogênico" (p. 58). Segundo os autores, mais crianças serão incluídas a partir da ampliação para a condição de espectro, o que traz a necessidade de problematizar esse aumento de diagnósticos, inclusive para a própria prática clínica do psicólogo que se deparará com uma demanda que abrange diversas possibilidades sintomáticas.

Nesse sentido, essas alterações de definição devem-se às consequentes revisões do DSM ao longo do tempo, além do rompimento com a psicanálise, sobretudo no ano de 1980, que representou o marco zero da nova relação entre psiquiatria e psicopatologia. Naquele ano, houve a publicação da terceira versão deste manual psiquiátrico que surgiu com novas proposições, ou seja, constituía-se agora de forma a ser a teórico e operacional (Dunker, 2014).

Segundo Russo eVenâncio (2006), com esta publicação houve rupturas em três níveis: 1) estrutura conceitual, ao propor uma única lógica classificatória; 2) abordagem psicanalítica, até então dominante nas edições anteriores; 3 ) representações sociais associadas ao indivíduo moderno, ao lidar com o normal e patológico e os grupos identitários. Todos esses níveis articulam-se entre si.

Essas rupturas, ocorridas na década de 1980, devem-se à pretensão psiquiátrica de ter a verdade do conhecimento sobre determinado objeto. Assim, teria que promover um sistema de classificação ideal que "deveria fornecer critérios explícitos, operacionalmente observáveis e que reduzissem ao mínimo o uso de interferências teóricas não diretamente observáveis para a definição de cada quadro mental" (Dunker \& Kyrillos Neto, 2015, p. 198).

Como afirmam Dunker e Kyrillos Neto (2011), entre a terceira e a quarta versão deste manual rompeu-se com a tradição presente desde Pinel. Nesta tradição, a caracterização do sofrimento e patologia mental era acompanhada da crítica filosófica. $\mathrm{O}$ perigo desse rompimento, como advertem os autores, é a adesão a práticas mecânicas de medicalização das massas. Isso acentua a importância de considerações a respeito de fenômenos como a suposta epidemia de autismo, posto que há uma implicação clínica que surge, sobretudo, frente a essas mudanças conceituais nos manuais diagnósticos.

Assim, esta publicação suscitou a exclusão dos debates teóricos, alçando-os para fora do campo psicopatológico. A justificativa é referida à "confusão de línguas" que existia em torno da psicopatologia, que impossibilitava a comunicação entre os diversos pesquisadores e clínicos. Para neutralizar essa confusão e tornar o manual operacional, procederam com a descrição minuciosa dos quadros. Contudo, apesar dessa soi-disant operacionalidade e neutralidade, a abordagem da terceira versão deste manual psiquiátrico nega que a escuta e o olhar são sempre condicionados pela dimensão subjetiva daquele que olha e escuta um sujeito (Pereira, 1996).

O transtorno, termo adotado pelo DSM, é também representante desta nova classificação. Traduzido para o português, corresponde ao original inglês disorder, isto é, algo fora da ordem. Nessa nomenclatura, segundo Pereira (1998), o transtorno efetuou um afastamento da linguagem nosográfica e foi declarado a teórico, de forma a escamotear "as questões políticas, sociais, culturais e subjetivas que afligem a vida humana" (Cirino, 2015, p. 32).

A pesquisa de López e Sarti (2013) realizada a respeito da assistência às crianças diagnosticadas com autismo expõe a fragilidade desse sistema que visa recolocar algo para dentro da ordem. Esta investigação etnográfica ocorreu em duas instituições públicas que recebiam autistas para tratamento: uma baseada na abordagem comportamental e outra amparada pela psicanálise. As autoras relatam que em um treinamento para o recebimento de novos estagiários na primeira instituição, a expositora apontava os ganhos que as crianças teriam ao participar de algumas atividades como montagens de blocos e mosaicos, e afirma ipsis litteris: "é importante saber que eles vão continuar chatos, esquisitos. .. mas a esquisitice passa mais despercebida, vão ficando mais próximos do normal" (López \& Sarti, 2013, p. 84).

A partir dessa fala e das outras observações, López e Sarti (2013) discutem sobre a tênue linha que separa o normal do patológico. Recorrem à Canguilhem e Foucault, a fim de elucidar a dinâmica pela qual a definição da patologia parte de uma complexa norma 
social que detém o poder para fazer essa delimitação. $\mathrm{O}$ que foge ao padrão na comparação das crianças diagnosticadas com autismo em relação às crianças ditas normais caracteriza o grau de comprometimento dentro do espectro.

A base que sustenta a fala da expositora de uma das instituições, relatada pelas autoras López e Sarti (2013), é a busca por aproximar a criança da normalidade. Ou seja, uma lógica oposta à que regula as intervenções psicanalíticas. Nessas intervenções, como se privilegia o singular, é impraticável participar de uma "lógica de normatização do sujeito" (Crespin, 2015, p. 395).

Esse artigo objetiva, justamente, tecer considerações a fim de situar em que campo ocorre esse aumento de crianças diagnosticadas com autismo. Assim, põe-se em relevo o que se passa nos campos psiquiátrico e social que fornecem esteio para que esse aumento ocorra. Observar o fenômeno sem as devidas considerações críticas gera o risco de se cair no previamente comentado, isto é, práticas baseadas em uma normatização que podem incorrer na medicalização e exclusão do sujeito de seu próprio sofrimento.

Em conformidade com o observado nesta investigação, Alfredo Jerusalinsky (2015) afirma que é neste ponto que se faz necessário pensar na subjetividade. Afinal, sem considerar o sujeito, estamos inclinados "a tomar os seres humanos como meros instrumentos de um projeto de produção do ideal" (p. 270).

Essa é uma posição semelhante também à de Julieta Jerusalinsky (2015). Segundo a autora, ao promover uma "patologização precoce", corre-se o risco de olhar a criança somente pela perspectiva do adoecimento. A partir de então, a referência do que se espera dela será apenas o prognóstico da doença. "Desse modo, o diagnóstico assume o valor preditivo do destino de um sujeito que amalgama o quadro nosográfico à dimensão ôntica do ser" (Jerusalinsky, 2015, p. 113).

Como transtorno característico da primeira infância, o autismo é circunscrito em um momento análogo de maturação cerebral, do organismo e de constituição psíquica. Não se trata aqui de expurgar o diagnóstico, mas admitir seus caminhos e descaminhos. Por um lado, o diagnóstico feito a tempo pode beneficiar a intervenção precoce. Por outro, há o risco de, se feito de modo fechado, beneficiar a colagem à patologia. Isso pode induzir que se estabeleça $\mathrm{a}$ criança em uma posição estática, fixada a traços patológicos, sem conceder espaço para a intervenção que analisa as possibilidades da construção subjetiva.

Essas são nuances de diagnósticos norteados por manuais psiquiátricos que ousam abordar os fenômenos humanos sem implicar a complexidade própria que essa condição nos impõe. A instabilidade, fruto desse sistema pragmático, foi exposta por meio da questão homossexual, de acordo com Pereira (2000).

Em 1970, diversos militantes gays invadiram a reunião da Associação Psiquiátrica Americana (APA) para lutar contra a patologização da homossexualidade. O combate político continuou, até que, em 1974, o termo "homossexualismo" é retirado do manual e passa a não ser mais considerado como categoria diagnóstica. A partir desse acontecimento, "as classificações supostamente científicas mostram sua grande vulnerabilidade face questões de poder, de interesses econômicos, de formação de grupos de pressão e de influência da mídia" (Pereira, 2000, p. 9).

Sobre esta fenda aberta na APA pela influência política dos militantes gays, Ortega (2008) aponta que no decorrer das últimas décadas, o estatuto nosológico de várias doenças psiquiátricas está sendo discutido em público. Segundo o autor, "embora não exista consenso sobre numerosas doenças psiquiátricas, o fato de serem nomeadas como doenças constitui uma forma de poder e utilidade social" (Ortega, 2008, p. 481).

A questão homossexual, que do estatuto de doença tornou-se um elo político-identitário, como apontam Russo e Venâncio (2006), se assemelha, em diversos aspectos, ao que tem começado a tomar forma com o autismo nos últimos anos. Com o advento da internet e as publicações dos autistas de alto funcionamento ${ }^{4}$, o movimento da neurodiversidade ganhou força. Com cada vez mais adeptos, estes passaram a problematizar a definição patológica do autismo. Para esse movimento, a pessoa não é autista, mas tem autismo, o que configura uma questão identitária.

Ortega (2008) estabelece que para o movimento da neurodiversidade o autismo é apenas uma conexão atípica do cérebro e não um transtorno mental que deva ser curado. Embora esse movimento posicione-se contra a psicanálise, Laurent (2014) expõe as aproximações que existem entre ambos, pois deveria

${ }^{4}$ Júnior, W. C. (2012, 2 de abril). Autistas de alto funcionamento apresentam nível cognitivo normal e não possuem atraso de fala. Que grau de autismo meu filho tem? Revista Autismo. http://www.revistaautismo.com.br/edicao-2/que-grau-de-autismo-meu-filho-tem 
interessá-los "a objeção psicanalítica a um tratamento comum, padronizado, dos sujeitos reunidos sob a mesma etiqueta" (p. 167).

\section{A perspectiva social: o diálogo entre patologia, associações de pais e identidade}

Em outro ponto de vista adjacente ao primeiro, o diagnóstico se funda e se sustenta socialmente, inclusive constituindo-se como importante lugar identitário. Isso é passível de ser observado, por exemplo, no protagonismo das associações de pais. Essas associações discorrem sobre a compreensão psiquiátrica do transtorno. Devido à mudança paradigmática operada com a publicação do DSM-III, foi possível a emergência dessas associações. De acordo com Ortega (2008), a separação do autismo do grupo das psicoses infantis e a inclusão nos Transtornos Invasivos do Desenvolvimento permitiram apagar a hipótese de uma psicogênese e, consequentemente, desimplicar pais e mães da causa do transtorno do filho.

O deslocamento do modelo psicanalítico, preponderante nas explicações etiológicas até então, foi em direção às explicações orgânicas, especialmente cerebrais. Afinal, "o cérebro responde cada vez mais por tudo aquilo que outrora nos acostumamos a atribuir à pessoa, ao indivíduo, ao sujeito" (Ortega, 2008, p. 490). Segundo o autor, esse afastamento da psicanálise e consequente aproximação de um paradigma cerebralista foi um dos fatores que permitiu ao autismo ser considerado como pertencente ao campo das deficiências.

A partir desse paradigma cerebral e cognitivo, estabelece-se outro posicionamento na compreensão do autismo. O rompimento com as concepções psicanalíticas direcionou as discussões para o seu estatuto orgânico, o que possibilitou que ele pudesse fincar seu locus na deficiência ${ }^{5}$. Diante da ausência de implicação dos pais na gênese do autismo dos filhos, abriram-se as portas para que esses se reunissem em associações que buscam lutar pelos direitos desses indivíduos. No Brasil, essa luta ocorre no campo da deficiência, lugar de assistência que o autismo ocupou historicamente no país (Oliveira, 2015).

Na perspectiva da deficiência, um caminho possível para o tratamento seria a reabilitação. Isto é, entende-se que é possível restituir habilidades àqueles que foram, de alguma forma, desabilitados. Como afirma Aranha (1995), os estudos sobre reabilitação, acelerados na Segunda Guerra Mundial, se sustentariam na possibilidade, então, de um reestabelecimento de prejuízos ou déficits ocasionados por uma lesão. Neste ponto de vista, há uma lesão no deficiente. Mas qual seria a lesão do autista?

Cabe pensar que a lesão que marca o autista ocorre na linguagem e na interação social. Sua reabilitação, então, deverá o restituir desse possível déficit. No entanto, não é essa a lógica que rege alguns programas de reabilitação a autistas, como constatam os estudos de Laurent (2014), López e Sarti (2013) e dos autistas de alto funcionamento Dawson (2004) e Bond (2012). Esses preferem o caminho do treinamento, da repetição e da adequação, em vista de uma possível funcionalidade do corpo e de uma melhor adaptação social. Entretanto, esse caminho despreza o que é marcadamente do campo do humano: as especificidades da constituição psíquica.

Este entrave entre a deficiência e o transtorno mental se desenvolve no campo das políticas públicas. Segundo Batista (2013), a deficiência e a loucura estiveram por longos anos entrelaçadas, com as teorias de Pinel e Itard. Somente com Esquirol rompe-se essa tradição, ao separar idiotia e loucura. Essa diferenciação ocorreu de modo que a primeira seria incurável, ao passo que para a segunda existia a possibilidade da cura. Além disso, a autora afirma que o uso da inteligência e a forma social de encarar o louco e o deficiente os diferem significativamente. O louco era visto de forma valorosa, como um predestinado, enquanto o deficiente reunia os aspectos monstruosos do humano.

Esse assombro com a deficiência ainda persiste devido ao estigma da organicidade e da incurabilidade. Entretanto, um amplo número de pais prefere compreender os filhos autistas como deficientes do que associá-los às psicoses infantis (Batista, 2013). Em outras palavras, travam um prélio epistemológico entre a compreensão do autismo como pertencente ao campo da deficiência ou do transtorno mental. Os efeitos de tais posicionamentos são severos em termos de políticas públicas, como nos mostra o caso do Brasil.

\footnotetext{
${ }^{5}$ Importante demarcar que é reconhecida, nesse trabalho, a heterogeneidade própria do campo da deficiência e suas relações com o autismo. No entanto, a opção por não trazer relatos mais pormenorizados justifica-se na medida em que a discussão privilegia outros aspectos sobre as associações de pais e a constituição de um campo narrativo e identitário dos autistas.
} 
No ano de 2013, o Ministério da Saúde/Brasil publicou duas cartilhas de assistência ao autismo, uma denominada "Diretrizes de Atenção à Reabilitação da Pessoa com Transtorno do Espectro Autista (TEA)" (Brasil, 2013a) e outra chamada "Linha de Cuidado para a Atenção às Pessoas com Transtornos do Espectro do Autismo e suas Famílias na Rede de Atenção Psicossocial do Sistema Único de Saúde" (Brasil, 2013b). Esses dois documentos, lançados pelo mesmo órgão e no mesmo ano, mas de orientações distintas, parecem evidenciar que há um desacordo no que se denomina como atenção à pessoa autista. O primeiro, intitulado de Diretrizes, está inserido na Rede de Cuidados da Pessoa com Deficiência, para o qual o tratamento adequado é a reabilitação. O segundo, intitulado como a Linha de Cuidado, situa o autismo na atenção psicossocial no campo dos transtornos mentais e sugere que $o$ atendimento aos autistas seja realizado na Rede de Atenção Psicossocial (RAPS) do SUS (Oliveira, 2015).

Oliveira (2015), em uma análise documental crítica desses dois documentos lançados pelo mesmo órgão competente, constatou que, embora o embate epistemológico seja decisivo nesse dissenso, não é o fator principal do conflito. O que se sobressai são controvérsias de diferentes grupos que disputam o protagonismo do poder de deliberação na formulação de políticas públicas para o autismo no Brasil.

O que antecede às construções desses documentos é que em termos de atenção ao autismo esse sempre se inseriu nos cuidados à deficiência mental. Lima, Couto, Delgado e Oliveira (2014) apontam que até o final do século passado essa população estava excluída da saúde pública brasileira e seu cuidado, quando ocorria, era prestado pelo campo filantrópico da educação ou assistência social. Coube às associações de pais montarem os primeiros serviços de assistência.

Novamente, o autismo configura-se em um campo dual de batalhas: deficiência ou transtorno. Nessa conjuntura, é importante problematizar a inclusão do autismo como deficiência, pois a criança que recebe esse diagnóstico tem direito ao benefício assistencial da Lei Orgânica de Assistência Social (Loas) ${ }^{6}$. Com base na lei, garante-se um salário mínimo mensal à pessoa que tenha idade acima de 65 anos ou que apresente alguma deficiência que o impossibilite de participar de forma plena e efetiva na sociedade. Para adquirir o benefício, é preciso que a renda por membro do grupo familiar seja inferior a um quarto do salário mínimo vigente.

Esse benefício pode ser um elemento externo que caracteriza para a família um lugar seguro e estável diante da instabilidade na convivência com um filho, muitas vezes, qualificado como estranho. Nessa medida, esses pais que se encontram sós diante daquilo que causa angústia no filho podem, às vezes, experimentar um sentimento de abandono, "que os leva a fazer do filho 'a causa' de sua vida e a militar a favor de seus direitos. O terceiro torna-se, então, puramente externo, reduzindo-se a ser aquele junto a quem cabe reivindicar mais direitos e mais cuidados" (Laurent, 2014, p. 32).

\section{Articulações entre a psiquiatria e o social: o problema da epidemia}

Os aspectos políticos apresentados anteriormente apontam para a união de pais em torno de associações para lutar pelos direitos de seus filhos diagnosticados com TEA. Atualmente, essas associações têm protagonizado papéis centrais na determinação de políticas de assistência e de atenção à criança com diagnóstico de autismo.

Como fortes dispositivos grupais, tais associações possibilitam a identificação entre pares e a procura por garantia de assistência e saúde a essa população que, como evidenciou Lima et al. (2014), esteve desassistida por bastante tempo. O reconhecimento do grupo como um dispositivo potente de cuidado tem fortalecido o movimento de grupos terapêuticos de pais em instituições. No campo psicanalítico, essas intervenções se guiam, sobretudo, pela escuta, que pode convocá-los a afastar-se da culpa, isolamento e exclusão que muitos vivenciam (Cavalcanti, 2000).

Essas associações também promovem um importante espaço grupal que permite aos pais identificações diversas. De acordo com Fortes (2009), na pós-modernidade há a vitória do gozo que exacerba o individualismo e enfraquece os vínculos afetivos, de maneira que

\footnotetext{
${ }^{6}$ Ministério do Trabalho e da Previdência Social. Benefício Assistencial ao Idoso e Pessoa com Deficiência. http://www.mtps.gov.br/servicos-do-ministerio/servicos-da-previdencia/beneficios-assistenciais-e-de-legislacao-especifica/beneficio-assistencial-ao-idoso-e-a-pessoa-com-deficiencia-bpc-loas
} 
vivemos em uma era de incertezas que mudou a relação do sujeito com as garantias quanto ao seu futuro. Isso conduz à sensação de vazio e de desproteção, à descrença na política, à fragilidade dos laços sociais e ao enfraquecimento da figura da alteridade nas nossas vidas (p. 1126).

De acordo com Bauman (2001), esta individualização constitui-se como uma marca pós-moderna. Nery e Vasconcellos (2014) argumentam que essa marca "representa o indivíduo que por si mesmo é capaz de construir sua própria trajetória e alcançar seus objetivos" (p. 124).

As associações de pais, reconhecidas em sua diversidade, parecem constituir-se para o indivíduo que participa como uma resistência ao que foi deflagrado no individualismo pós-moderno e está posto nos dias de hoje. Forma-se, assim, uma comunidade que possui e luta pelos mesmos objetivos. Interessante notar que isso aconteça contemporaneamente sobretudo pela via da patologia. Isto é, há comunidades que se desenvolvem a partir de uma nomenclatura diagnóstica.

A partir disso, além de pensar no espaço grupal ofertado pelas associações de pais, seria possível também sinalizar que há algo no diagnóstico que escapa à medicina e à psiquiatria. Este não se refere mais a apenas seu objetivo técnico de identificar e tratar uma doença. O diagnóstico oferta um lugar social, promotor de identificações, que cumpre uma função psíquica.

Evidencia-se, assim, o caráter social e subjetivo que permeia a psiquiatria e que esta tenta a todo custo evitar. Restrita aos manuais a teóricos, este ramo da psiquiatria contemporânea, biologicista, tenta atribuir uma neutralidade impossível de existir em termos de psicopatologia. Não é possível abordar uma articulação entre a perspectiva psiquiátrica e social dos transtornos mentais, na medida em que a primeira se instaura sob a égide da segunda. Dessa forma, o que se produz no campo psiquiátrico atualmente é fruto do que se passa socialmente na contemporaneidade.

O debate torna-se ainda mais interessante ao problematizarmos a questão da suposta epidemia do TEA, que de transtorno bastante raro e quase desconhecido tem se popularizado enormemente - como denotam as pesquisas recentes (Ortega et al., 2013;
Rios et al., 2015). Se reconhecemos o individualismo como um importante aspecto pós-moderno, é possível conjecturar que há um aumento de traços autísticos posto no próprio laço social contemporâneo.

Para tanto, parece que a epidemia de autismo se vincula a isso que se passa entre os campos psiquiátrico e social atualmente. Essa epidemia, edificada na era pós-moderna, aponta para um interessante paradoxo. O individualismo exacerbado nesta era encontra oposição pela via da patologia. Unidos por uma nomenclatura diagnóstica, é possível formar vínculos e pertencer a um grupo.

\section{Considerações finais}

A questão colocada sobre uma possível epidemia de autismo parece ser facilmente respondida pelos dados epidemiológicos. Há que se reconhecer o crescimento diagnóstico vertiginoso dos últimos anos: de sintoma da esquizofrenia infantil, o autismo galgou patamares até atingir nomenclatura própria, englobando diversas outras manifestações sintomáticas da infância descrita nos manuais psiquiátricos anteriores, como a Síndrome de Asperger e o Transtorno Desintegrativo da Infância.

Contudo, apenas a vertente psiquiátrica parece insuficiente para explicar a popularização do transtorno nos últimos anos. O campo social desempenhou papel importante nessa popularização e consequente crença sobre a epidemia. Nesse sentido, é no que se refere ao campo social que interessa pensar os efeitos desse aumento expressivo de crianças diagnosticadas com TEA. Isso implica que haja escuta e investimento para famílias que sofrem e se angustiam com o sintoma de suas crianças, assim como para professores e outros profissionais que serão convocados a pensar o desafio referido à inclusão.

Em termos clínicos, os diagnósticos psiquiátricos na infância expõem demandas que sugerem um posicionamento ético do psicólogo, isto é, aquele que escuta o sofrimento do sujeito. Há que se propor a operar, sobretudo, uma clínica que seja mediada pelo cuidado e pela leitura atenta de fenômenos complexos e sutis que envolvem os aspectos de saúde mental e que perpassam um momento tão específico da vida do sujeito como a infância. 


\section{Referências}

American Psychiatric Association. (1952). Diagnostic and Statistical Manual of Mental Disorders - DSM-I. Washington, DC: APA.

American Psychiatric Association. (1968). Diagnostic and Statistical Manual of Mental Disorders - DSM-II(2nd ed.). Washington, DC: APA.

American Psychiatric Association. (1980). Diagnostic and Statistical Manual of Mental Disorders - DSM-III (3rd ed.). Washington, DC: APA.

American Psychiatric Association. (1994). Manual diagnóstico e estatístico de transtornos mentais - DSM-IV (4a ed.). Porto Alegre, RS: Artes Médicas.

American Psychiatric Association. (2013). Manual diagnóstico e estatístico de transtornos mentais (5a ed.). Porto Alegre, RS: Artes Médicas.

Aranha, M. S. F. (1995) Integração social do deficiente: análise conceitual e metodológica. Temas em Psicologia, 3(2), 63-70.

Araújo, R. R., Souza-Silva, J. R., \& D’Antino, M. E. (2012). Breve discussão sobre o impacto de se ter um irmão com transtorno do espectro do autismo. Cadernos de Pós-Graduação em Distúrbios do Desenvolvimento, 12(1), 9-15.

Batista, C. A. M. (2013, 8 de julho). Deficiência e autismo [blog]. https://psicanaliseautismoesaudepublica. wordpress.com/2013/07/08/deficiencia-e-autismo-cristina-abranches-mota-batista/

Bauman, Z. (2001). Modernidade líquida. Rio de Janeiro, RJ: Zahar.

Bertrand, J., Mars, A., Boyle, C., Bove, F., \& Yeargin-Allsopp, M. (2001). Prevalence of autism in a United States population: The Brick Township, New Jersey, investigation. Pediatrics, 108(5), 1155-1161.

Bond, H. (2012, April 16). What autism can teach us about psychoanalysis. The Guardian. https://www.theguardian.com/commentisfree/2012/apr/16/autism-psychoanalysis-lacanian

Brasil. (2013a). Diretrizes de Atenção à Reabilitação da Pessoa com Transtorno do Espectro Autista (TEA). Brasília, DF: Ministério da Saúde. http://bvsms.saude.gov.br/bvs/publicacoes/diretrizes_atencao_reabilitacao_pessoa_ autismo.pdf

Brasil. (2013b). Linha de cuidado para a atenção às pessoas com transtorno do espectro do autismo e suas famílias na Rede de Atenção Psicossocial do Sistema Único de Saúde. Brasília, DF: Ministério da Saúde http://bvsms.saude. gov.br/bvs/publicacoes/linha_cuidado_atencao_pessoas_transtorno.pdf

Bursztejn, C. (2016). Evolução dos conceitos de autismo e psicose na criança. In M. C. Laznik, B. Touati \& C. Bursztejn (Orgs.), Distinção clínica e teórica entre autismo e psicose na infância (pp. 9-26). São Paulo, SP: Instituto Langage.

Cavalcanti, A. E. (2000). Novas narrativas para os autismos: algumas contribuições de Winnicott para uma teoria e clínica dos autismos. Estilos da Clínica, 5(8), 38-48.

Center for Disease Control and Prevention. (2010). Identified prevalence of autism spectrum disorder. http://www.cdc.gov/ncbddd/autism/data.html

Center for Disease Control and Prevention. (2014). Prevalence of autism spectrum disorders among children aged 8 years: autism and developmental disabilities monitoring network, 11 sites, United States, 2010. Surveillance Summaries, 63(2), 1-21.

Charman, T. (2002). The prevalence of autism spectrum disorders: recent evidence and future challenges. European Child and Adolescent Psyquiatry, 11(6), 249-256.

Cirino, O. (2015). Genealogia da psiquiatria da infância. In M. Kamers, R. M. Mariotto, \& R. Voltolini (Orgs.), Por uma (nova) psicopatologia da infância e adolescência (pp. 19-40). São Paulo, SP: Escuta.

Crespin, G. (2015). Como trabalha um psicanalista com uma criança autista sem linguagem? In M. C. Kupfer \& M. Szejer (Orgs.), Luzes sobre a clínica e o desenvolvimento de bebês: novas pesquisas, saberes e intervenções (pp. 387-410). São Paulo, SP: Instituto Langage.

Dawson, M. (2004, 18 de janeiro). The misbehaviour of behaviourists. Ethical Challenges to the autism ABA industry. http://www.sentex.net/ nexus23/naa_aba.html 
Dunker, C. I. L. (2014). Questões entre a psicanálise e o DSM. Jornal de Psicanálise, 47(87), 79-107.

Dunker, C. I. L., \& Neto, F. K. (2011). A crítica psicanalítica do DSM-IV: breve história do casamento psicopatológico entre psicanálise e psiquiatria. Revista Latinoamericana de Psicopatologia Fundamental, 14(4), 611-626.

Dunker, C. I. L., \& Kyrillos Neto, F. (2015). Psicanálise e saúde mental. Porto Alegre, RS: Criação Humana.

Fávero, M. A. B., \& Santos, M. A. (2005). Autismo infantil e estresse familiar: uma revisão sistemática da literatura. Psicologia: Reflexão e Crítica, 18(3), 358-369.

Fombonne, E. (1999). The epidemiology of autism: a review. Psychological Medicine, 29, 769-786.

Fombonne, E. (2003). The prevalence of autism. JAMA, 289(1), 87-89.

Fombonne, E. (2009). Epidemiology of pervasive developmental disorders. Pediatric Research, 65(1), 591-598.

Fortes, I. (2009). A psicanálise face ao hedonismo contemporâneo. Revista Mal-Estar e Subjetividade, 9(4), 1123-1144.

Gomes, P. T. M., Lima, L. H. L., Bueno, M. K. G., Araújo, L. A., \& Souza, N. M. (2015). Autismo no Brasil, desafios familiares e estratégias de superação: revisão sistemática. Jornal de Pediatria, 91(2), 111-121.

Jerusalinsky, A. (2015). Para um autista uma verdadeira inclusão acontece quando ele decide se incluir. In A. Jerusalinsky (Org.), Dossiê autismo (pp. 252-275). São Paulo, SP: Instituto Langage.

Jerusalinsky, J. (2015). Detecção precoce de sofrimento e psicopatologia na primeira infância: a desobediência dos bebês aos critérios nosográficos deve ser considerada. In M. Kamers, R. M. Mariotto, \& R. Voltolini (Orgs.), Por uma (nova) psicopatologia da infância e da adolescência (pp. 103-116). São Paulo, SP: Escuta.

Kanner, L. (1997). Os distúrbios autísticos do contato afetivo. In P. Rocha (Org.), Autismos (pp. 111-170). São Paulo, SP: Escuta. (Trabalho original publicado em 1943)

Klin, A. (2006). Autismo e síndrome de Asperger: uma visão geral. Revista Brasileira de Psiquiatria, 28(1), 3-11.

Laurent, E. (2014). A batalha do autismo: da clínica à política. Rio de Janeiro, RJ: Escuta.

Lima, R. C., Couto, M. C. V., Delgado, P. G. G., \& Oliveira, B. D. C. (2014). Indicadores sobre o cuidado a crianças e adolescentes com autismo na rede de CAPSi da região metropolitana do Rio de Janeiro. Physis, 24(3), 715-739.

López, R. M. M., \& Sarti, C. (2013). Eles vão ficando mais próximos do normal... Considerações sobre normalização na assistência ao autismo infantil. Idéias, 6(1), 77-98.

Nery, A. D., \& Vasconcellos, E. G. (2014). Individualização e fragmentação: efeitos da pós-modernidade no cristianismo contemporâneo. Ciências da Religião: História e Sociedade, 12(2), 118-132.

Oliveira, B. D. C. (2015). Análise das políticas públicas brasileiras para o autismo: entre a atenção psicossocial e a reabilitação (Dissertação de mestrado). Universidade do Estado do Rio de Janeiro, Rio de Janeiro, RJ.

Ortega, F. (2008). O sujeito cerebral e a neurodiversidade. Mana, 14(2), 477-509.

Ortega, F., Zorzanelli, R., Meierhoff, L. K., Rosário, C. A, Almeida, C. F., Andrada, B. F. C. C., Chagas, B. S., \& Feldman, C. (2013). A construção do diagnóstico de autismo em uma rede social virtual brasileira. Interface, 17(44), 119-132.

Paula, C. S., Fombonne, E., Gadia, C., Tuchman, R., \& Rosanoff, M. (2011). Autism in Brazil:- perspectives from science and society. Revista da Associação Médica Brasileira, 57(1), 2-5.

Pereira, M. E. C. (1996). Questões preliminares para um debate entre a psicanálise e a psiquiatria no campo da psicopatologia. In L. F. S. Couto, Pesquisa em Psicanálise (pp. 43-54). Belo Horizonte, MG: Segrac.

Pereira, M. E. C. (1998). O DSM-IV e o objeto da psicopatologia ou psicopatologia para quê? www.estadosgerais.org/ historia/98-dsm-iv.shtml

Pereira, M. E. C. (2000). A paixão nos tempos do DSM: sobre o recorte operacional do campo da psicopatologia. In R. Pacheco Filho et al., Ciência, pesquisa, representação em psicanálise (pp. 119-152). São Paulo, SP: Casa do Psicólogo.

Ribeiro, S. H. B. (2007). Prevalência dos transtornos invasivos do desenvolvimento no munícipio de Atibaia: um estudo piloto (Dissertação de mestrado). Universidade Presbiteriana Mackenzie, São Paulo, SP.

Rice, C. E., Rosanoff, M., Dawson, G., Durkin, M. S., Croen, L. A., Singer, A., \& Yeargin-Allsopp, M. (2012). Evaluating changes in the prevalence of the autism spectrum disorders. Public Health Reviews, 34(2), 2-22. 
Rios, C., Ortega, F., Zorzanelli, R., \& Nascimento, L. F. (2015). Da invisibilidade à epidemia: a construção narrativa do autismo na mídia impressa brasileira. Interface, 19(53), 325-335.

Russo, J., \& Venâncio, A. T. A. (2006). Classificando as pessoas e suas perturbações: a revolução terminológica do DSM III. Revista Latinoamericana de Psicopatologia Fundamental, 9(3), 460-483.

Rutter, M. (2004). Incidence of Autistic spectrum disorders: changes over time and their meaning. Acta Pediatrica, 94, 2-15.

Touati, B., Mercier, A., \& Tuil, L. (2016). Autismo, uma pesquisa. Da necessidade de reprecisar o campo do autismo e aqueles dos TID não autístico. In M. C. Laznik, B. Touati, \& C. Bursztejn (Orgs.), Distinção clínica e teórica entre autismo e psicose na infância (pp. 57-90). São Paulo, SP: Instituto Langage.

\section{Maíra Lopes Almeida}

Mestre em Psicologia na linha de "Psicanálise e Cultura" pela Universidade Federal de Uberlândia, Uberlândia MG. Brasil

E-mail: maira.lpalmeida@gmail.com

(D) https://orcid.org/0000-0002-6956-9858

\section{Anamaria Silva Neves}

Doutora em Psicologia pela Universidade de São Paulo (USP). Pós-doutorado pelo Child and Woman Abuse Studies Unit (CWASU) da London Metropolitan University. Docente do curso de Psicologia da Universidade Federal de Uberlândia, Uberlândia - MG. Brasil

E-mail: anamaria.neves@ufu.br

(D) https://orcid.org/0000-0002-7722-8690

Endereço para envio de correspondência:

Universidade Federal de Uberlândia, Instituto de Psicologia, Campus Umuarama. Av. Pará, 1.720, Bloco 2C. CEP: 38450-320. Uberlândia - MG. Brasil.

Recebido 01/06/2016

Aceito 03/07/2018

Received 01/06/2016

Approved 03/07/2018

Recibido 01/06/2016

Aceptado 03/07/2018

Como citar: Almeida, M. L., \& Neves, A. S. (2020). A Popularização Diagnóstica do Autismo: uma Falsa Epidemia? Psicologia: Ciência e Profissão, 40, 1-12. https://doi.org/10.1590/1982-3703003180896

How to cite: Almeida, M. L., \& Neves, A. S. (2020). The Diagnostic Popularization of Autism: a False Epidemic? Psicologia: Ciência e Profissão, 40, 1-12. https://doi.org/10.1590/1982-3703003180896

Cómo citar: Almeida, M. L., \& Neves, A. S. (2020). La popularización diagnóstica del autismo: una falsa epidemia? Psicologia: Ciência e Profissão, 40, 1-12. https://doi.org/10.1590/1982-3703003180896 